\title{
Zur Frage der Glykogenbildung aus Fettstoffen, insbesondere Fettsäuren unter Adrenalinwirkung.
}

\author{
Von \\ Kunizo Sanzyo. \\ (三條 國藏) \\ (Aus der Medizinischen Klinik von Prof. S. Yamakawa, an \\ der. Tohoku Kaiserlichen Universität zu Sendai.)
}

\section{Einleitung.}

Obwohl der Übergang von Kohlehydraten in Fette schon z. B aus den Erfahrungen der Tierzüchter; welche gewöhnlich durch forcierte Kohlehydratfütterung reichlichen Fettansatz erzielen, ohne weiteres angenommen wird, steht man dem umgekehrten Vorgang, der Zuckerbildung aus Fetten, trotz mancher anscheinend dafür sprechenden Ergebnisse der vorangehenden Autoren noch immer skeptisch gegenüber. Das hängt zum Teil mit einer anderen ebenso wenig gelösten Frage zusammen, ob das Fett physiologischerweise direkt als solches oder erst auf dem Weg der Kohlehydrate im Körper oxydiert wird.

Manche Forscher, die die Zuckerbildung aus Fett bestätigt zu haben glauben, nahmen ihre Untersuchungen stets unter den pathologischen Bedingungen vor, bei denen der Kohlehydratstoffwechsel der durch Hunger, Strychninkrämpfe, Phloridzin- oder Thyreoideaverabreichung glykogenarm gemachten Tiere durch Zufuhr von Adrenalin oder Nebennierenextrakt zwangmässig gesteigert war. Bei so vorbehandelten Tieren erzeugte die Fettfütterung bald eine Blutzuckererhöhung (Asher u. Clavo-Criado, ${ }^{1)}$ Bickenbach u. Junkersdorf ${ }^{2)}$ ) bald eine vermehrte Glykosurie (Blum, ${ }^{3)}$ Velich, ${ }^{4)}$ Eppinger, Falta u. Rudinger, ${ }^{5)}$ Wertheim er ${ }^{6}$ ), oder endlich eine deutliche Glykogenablagerung (Pollak, ${ }^{7}$ Roubitschek, ${ }^{8}$ ) Burn u. Marks, ${ }^{9}$ ( Ohara, ${ }^{10}$ Matsui, ${ }^{11)}$ Aok $i^{12)}$ ). Mit diesen Ergebnissen soll man jedoch nach der Meinung von v. Fürth ${ }^{13)}$ die These von der Zuckerbildung aus Fett keineswegs als unumstösslich betrachten, 
wenigstens aus den Fettsäurekomponenten des Fettes, denn aus den Glyzerinkomponenten kann, wie man allgemein annimmt, eine wenn auch kleine Menge Zucker gebildet werden. In hiesiger Klinik wurde auch diese Frage schon seit langem von mehreren Forschern eifrig bearbeitet. Unter anderen konnte $\mathrm{Kimura}{ }^{14)}$ unter Thyroxin- and A oki unter Adrenalinwirkung in der glykogenarm gemachten Leber Zuckerbildung aus intravenös eingespritztem, emulgiertem Fett feststellen. Da aber diese Untersuchungen auch nichts iber die Zuckerbildung aus den Fettsäurekomponenten zu Tage fördern, so habe ich dasselbe Thema übernommen, indem ich nachzuweisen versuche, ob bei den durch Adrenalin zur Stoffwechselsteigerung gereizten Tieren Glykogenstapelung im Anschluss an die perorale Fett- und insbesondere Fettsäureverabreichung erzielt werden könne.

\section{Resorption der Fette und Fettsäuren aus dem Darm.}

Da im vorliegenden Versuch Fett und Fettsäure als Quelle der Glykogenneubildung peroral eingefiuhrt werden, so muss man sich vor allem darüber vergewissern, wie die Resorption derart verabreichter Substanzen vor sich geht. Zur Untersuchung wurden zunächst die Kaninchen, die man seit 3 Tagen, um die im Darmschlauch anwesenden Nahrungsreste möglichst zu beseitigen, hat hungern lassen, laparotomiert und eine Strecke des. Darmrohrs von dem übrigen isoliert, indem man den Darm an zwei Stellen, nämlich dicht unterhalb des Pylorus und ca. $50 \mathrm{~cm}$ weit distal davon durch Ligatur fest abschnirte, wobei natürlich die $Z$ wölffingerdarmpapille unversehrt in der obersten Partie des so hergestellten Darmsacks aufsitzt. Eine Menge Olivenöl resp. Oleinsäure wurde mit einem Zehntel Gewichtsteil Rindergalle im Mörser gründlich verrieben und durcheinander gemischt. Die so hergestellte Mischung, deren Gehalt an Ätherextrakt vorläufig nach $\mathrm{K} u \mathrm{magaw}$ a-Su $\mathrm{to}^{15}$ ) bestimmt worden war, wurde in einer $4 \mathrm{~g}$ Ätherextrakt entsprechenden Menge pro Tier ins obere Ende des blinden Darmrohrs mittels Spritze injiziert und die Bauchdecke geschlossen, um den Darmsack in situ zu eihalten, bis nach einem Zeitlauf der Darmsack herausgeschnitten, dessen Inhalt dann zusammen mit den wiederholt berieselten Spülflüssigkeiten quantitativ abgefangen und mittels des Kumagawa-Sutoschen Flüssigkeitsextraktors mit Äther gründlich extrahiert wurde. Der Ätherextrakt wurde auf dem Wasserbad verdunstet, der getrocknete Rückstand alsdann in reinem 
Äther gelöst. Der durch wiederholte Extraktion gereinigte Ätherextrakt wurde in den Tabellen als der wiedergefundene, der Resorption entgehende Teil des eingespritzten Fettes bezw. der Fettsäure angegeben. Tab. 1 gibt die Geschwindigkeit der Resorption des Olivenöls

Tabelle 1.

Resorption des Olivenöls aus dem isolierten Darmschlauch.

\begin{tabular}{|c|c|c|c|c|c|c|}
\hline \multirow[b]{2}{*}{ Datum } & \multirow[b]{2}{*}{$\begin{array}{c}\text { Versuchs- } \\
\text { tier-Nr. }\end{array}$} & \multirow{2}{*}{$\begin{array}{l}\text { Körper- } \\
\text { gewicht } \\
\text { (g) }\end{array}$} & \multirow{2}{*}{$\begin{array}{l}\text { Zeitdauer } \\
\text { zur Re- } \\
\text { sorption } \\
\text { (Std.) }\end{array}$} & \multicolumn{3}{|c|}{ Olivenöl } \\
\hline & & & & $\begin{array}{c}\text { ein- } \\
\text { geführt } \\
(\mathrm{g})\end{array}$ & $\begin{array}{l}\text { wieder- } \\
\text { gefunden } \\
\text { (g) }\end{array}$ & $\begin{array}{c}\text { resorbiert } \\
(\%)\end{array}$ \\
\hline 2/III '37 & 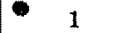 & 1880 & 1 & 4,00 & 3,86 & 3,5 \\
\hline $3 / \mathrm{III}$ & 2 & 1600 & 1 & 4,00 & 3,81 & 4,8 \\
\hline Durohschnitt & & & & & & 4,1 \\
\hline 2/III & 3 & 1450 & $\mathbf{3}$ & 4,00 & 3,24 & 19,0 \\
\hline $3 / \mathrm{III}$ & 4 & 1450 & 3 & 4,00 & 3,41 & 14,7 \\
\hline Durchsohnitt & & & & & & 16,8 \\
\hline 3/III & 5 & 1800 & 5 & 4,00 & 2,98 & 25,5 \\
\hline $10 / \mathrm{III}$ & 6 & 1480 & 5 & 4,00 & 3,12 & 22,0 \\
\hline Durchschnitt & & & & & & 23,7 \\
\hline $10 / \mathrm{III}$ & $\mathbf{7}$ & 1460 & 7 & 4,00 & 2,91 & 27,2 \\
\hline $12 / \mathrm{III}$ & 8 & 1550 & 7 & 4,00 & 3,04 & 24,0 \\
\hline Durchschnitt & & & & & & 25,6 \\
\hline
\end{tabular}

aus dem isolierten Darmsack wieder. Innerhalb einer Stunde findet die Resorption des Neutralfettes nur ganz gering statt. Erst nach 3 Stunden beobachtet man eine Resorption um 16,8\%, nach 5 Stunden eine um $23,7 \%$, und schliesslich nach 7 Stunden eine um 25,6\% des eingeführten Fettes. Etwas schneller geht die Resorption der Fettsäure vor sich, wie aus Tab. 2 hervorgeht. Bei dieser sieht man eine

Tabelle 2.

Resorption der Oleinsüure aus dem isolierten Darmschlauch.

\begin{tabular}{|c|c|c|c|c|c|c|}
\hline \multirow[b]{2}{*}{ Datum } & \multirow[b]{2}{*}{$\begin{array}{c}\text { Versuchs- } \\
\text { tier-Nr. }\end{array}$} & \multirow{2}{*}{$\begin{array}{l}\text { Körper- } \\
\text { gewicht } \\
\text { (g) }\end{array}$} & \multirow{2}{*}{$\begin{array}{l}\text { Zeitdauer } \\
\text { zur Re- } \\
\text { sorption } \\
\text { (Std.) }\end{array}$} & \multicolumn{3}{|c|}{ Oleinsänre } \\
\hline & & & & $\begin{array}{c}\text { ein- } \\
\text { gefinht } \\
(\mathbf{g})\end{array}$ & $\begin{array}{c}\text { wieder- } \\
\text { getunden } \\
\text { (g) }\end{array}$ & $\begin{array}{c}\text { resorbiert } \\
(\%)\end{array}$ \\
\hline $5 / 111$ ' 37 & 9 & $1450^{\circ}$ & 1 & 4,01 & 3,56 & 11,2 \\
\hline $\begin{array}{l}\text { 10/III } \\
\text { Durchschnitt }\end{array}$ & 10 & 1780 & 1 & 4,01 & 3,75 & $\begin{array}{l}6,4 \\
8,8\end{array}$ \\
\hline 5/III & 11 & 1800 & 3 & 4,01 & 2,54 & 36,6 \\
\hline 10/III & 12 & 1600 & 3 & 4,01 & 2,89 & $\begin{array}{l}27,9 \\
32,2\end{array}$ \\
\hline $\begin{array}{l}\text { Durehsennitt } \\
\text { 5/III }\end{array}$ & 13 & 1700 & 5 & 4,01 & 2,72 & 32,2 \\
\hline $\begin{array}{l}\text { 10/III } \\
\text { Durehsehnitt }\end{array}$ & 14 & 1580 & 5 & 4,01 & 2,43 & $\begin{array}{l}39,4 \\
\mathbf{3 5}, 8\end{array}$ \\
\hline
\end{tabular}


Resorption um 8,8\% nach einer Stunde, eine um $32,2 \%$ nach 3 Stunden und eine um $35,8 \%$ nach 5 Stunden.

Dass das Fett vornehmlich im Dünndarm resorbiert wird, ist schon allgemein bekannt (Cl. Bernard, London $u$. Wersilow $a^{16)} u$. s. w.). Die Geschwindigkeit der Fettresorption hängt nicht unwesent lich von der Zersetzlichkeit und Emulgierbarkeit der Substanzen ab, und es ist von Arnschink ${ }^{17)}$ und Levite ${ }^{18)}$ festgestellt, dass die an ungesättigten Ketten reichen Fettarten, die von tieferen Schmelzpunkten sind, im allgemeinen leichter resorbierbar sind als die anderen. Von diesem Gesichtspunkt betrachtet müssen Olivenöl und Oleinsäure, die in dieser Arbeit ausschliesslich gebraucht wurden, eine am leichtesten resorbierbare Fettart darstellen. Gewiss konnten Munk und Rosenste in ${ }^{19)}$ zeigen, dass Olivenöl schon 2 Stunden nach der Abgabe im Lymph erscheint und nach 5 Stunden das Resorptionsmaximum erreicht. Im Gegensatz zu Neutralfett sollen aber nach Stra c ${ }^{20}$ die freien Fettsäuren nur in einer sehr geringen Menge aus dem Darm resorbiert werden, obwohl die Neutralfette nach Meinungen von Pflüg e ${ }^{21)}$ und anderen erst nach der Spaltung zur Resorption kommen. Die Beobachtung Stracks stimmt aber mit meinem Ergebnis nicht überein, bei welchem Oleinsäure eher etwas besser als Olivenöl resorbiert wurde. Natürlich kann das tiefgreifende Versuchsverfahren nicht unwesentlich das physiologische Resorptionsverhältnis geändert haben.

\section{Glykogenbildung aus Fett und Fettsäure in normaler Leber unter Adrenalinwirkung.}

\section{a) Versuchsmethode.}

Zur Untersuchung wurden atasschliesslich reife männliche Kaninchen genommen, die man seit 24 Stunden hat hungern lassen. Den Tieren, die vor einer halben Stunde $2 \mathrm{ccm}$ Opiumtinktur zum Vorbeugen der Diarrhoe erhalten hatten, wurde eine Menge von Olivenöl resp. Oleinsäure, die wie früher erwähnt durch Zusatz der Rindergalle emulgiert worden war, mittels einer Sonde in den Magen eingeführt. Am Ende der Versuche wurde die Leber aus den durch Luftembolie getöteten Tieren schnell herausgenommen und deren Glykogengehalt durch die in dieser Klinik modifizierte Bierrysche Methode, ${ }^{22}$ und Fettgehalt nach Verseifungsmethode von $\mathrm{Ku} \mathrm{magawa-Suto} \mathrm{be-}$ stimmt. Adrenalin wurde stets subkutan appliziert. Um die individuellen. Schwankungen auszugleichen, zog man stets 3 Tiere zur Paralleluntersuchung heran, wobei aber diejenigen, die trotz der vorbeugenden Opiumanwendung mit mehr oder minder starker Diarrhoe einhergingen, aus der Erwägung der Ergebnisse ausgeschlossen wurden. 


\section{b) Kontrollwert für Glykogen-und Fettgehalt der. Leber bei Normaltieren.}

Zuerst wurde als Kontrollversuch der Glykogen- und Fettgehalt der Kaninchen ermittelt, die seit 24 Stunden gehungert hatten und nur mit Galle und Opiumtinktur von gleichen Mengen wie in den

Tabelle 3.

Glyjkogen- und Fettgehalt der Leber der Normalkaninchen.

11.-12. Jan. 1937.

\begin{tabular}{c|c|c|c|c}
\hline $\begin{array}{c}\text { Versuchstier- } \\
\text { Nr. }\end{array}$ & $\begin{array}{c}\text { Körpergewicht } \\
(\mathrm{g})\end{array}$ & \multicolumn{3}{|c|}{ Leber } \\
\cline { 3 - 5 } & & Gewieht (g) & Glykogen (\%) & Fett $(\%)$ \\
\hline & 1620 & 51 & 1,78 & \\
16 & 1530 & $\mathbf{4 4}$ & 1,58 & 3,03 \\
17 & 1710 & 53 & 1,64 & $\mathbf{2 , 9 5}$ \\
Durchschnitt & & & $\mathbf{1 , 0 6}$ & $\mathbf{3 , 0 2}$ \\
& & &
\end{tabular}

Hauptversuchen beschickt wurden, aber keine Fettsubstanzen und Adrenalin erhielten. Der Versuch wurde wie die meisten anderen Hauptversuche in der kalten Jahreszeit vorgenommen. Als Kontrollwert ergibt sich daraus für Glykogen 1,66\% und für Fett 3,02\%.

\section{c) Wirkung des Adrenalins auf den Glykogen- und Fettgehalt der Leber der Normalticre.}

Adrenalin ruft bekanntlich Hyperglykämie und manchmal auch Glykosurie hervor, als deren Quelle natürlich das Leberglykogen die Hauptrolle spielen muss (Doyon u. Kareff, ${ }^{23)}$ Wolownik, ${ }^{24}$ ) Bang ${ }^{26)}$ ), wenn aber auch das Muskelglykogen (C. F. Cori und G. T. Cori, ${ }^{26)}$ Geiger u. Schmidt, ${ }^{27)}$ Corkillu. Marks s${ }^{28}$ ) und sogar auch das Fett (Velich,4) Wertheimer6) daran beschuldigt wird. Jedenfalls reagiert die Leber stets auf Adrenalin anfangs mit Verminderung des Depotsglykogens, das aber später allmählich wiederhergestellt wird (Bierry u. Gruzewska, ${ }^{29)}$ Molitor, ${ }^{30}$ ) Cori, ${ }^{31}$ ) Sacks, ${ }^{32)}$ Young ${ }^{33)}$ ): Diese nachträgliche Anhäufung des. Vorratglykogens tritt nach Untersuchungen von Sahyun u. Luck, ${ }^{34}$ ) Cori, Cori und Buchwald ${ }^{35)}$ und Nakazuka ${ }^{36)} 3$ oder 5 Stunden nach der Adrenalinverabreichung ein und besteht mehrere Stunden lang fort. Löw und Pfeile $\mathrm{r}^{37)}$ geben an, dass die Leber auf Adrenalin je nach dem Ernährungszustand verschieden reagiert und zwar, 
falls sie mit normalem Glykogengehalt versehen ist, schüttet sie einen Teil des letzteren aus, dagegen, wenn sie daran sehr arm ist, z. B. infolge des Hungerns, gewinnen die Depots mehr oder minder reichlich. A o ki konnte schon früher in dieser Klinik zeigen, dass der Glykogengehalt der Leber der Kaninchen, die im voraus Fettemulsion intravenös erhielten, 5 Stunden nach Adrenalin bedeutend vermindert, dagegen 8 Stunden danach wieder etwas und nach 12 Stunden noch stärker vermehrt wird. Die Ergebnisse der vorangehenden Autoren geben mir den Eindruck, dass man in einem derartigen Versuch gewöhnlich gegen 12 Stunden nach der Adrenalineinspritzung die besten Bedingungen erzielen kann, um aus einer Muttersubstanz gebildetes Glykogen als Ausbeute der Leberanalyse nachzuweisen. In diesen späteren Stunden der Adrenalinwirkung werden wahrscheinlich die Tierkörper so umgestimmt, aus irgendwelchen Muttersubstanzen Glykogen neuzubilden und teilweise in den Depots aufzubewahren. Somit habe ich bei dieser Arbeit, die beabsichtigt war, die Glykogenausbeute aus Fettsubstanzen zu bestimmen, die Tiere stets 12 Stunden nach der Adrenalineinspritzung getötet. Als Kontrolle wurde ein Versuch vorausgeschickt, bei dem nur verschiedene Mengen Adrenalin eingespritzt wurden, ohne aber zu prüfende Fettsubstanzen zu geben (Tab. 4-6). Bei Anwendung von 0,2 ccm Adrenalinlösung (Tab. 4) erhielt man durchschnittlich 1,71\% Leberglykogen, die ein bisschen grösser sind als der Normalwert von 1,66\%, bei der Giftdosis von $0,5 \mathrm{ccm} 1,51 \%$ Leberglykogen, die also etwas kleiner sind als letzterer. Bei diesen verhältnismässig kleineren Adrenalinmengen müssen die anfangs durch Giftwirkung hervorgerufenen Senkungen des Vorratglykogens natürlich nicht so gross gewesen und deswegen um diese spätere Zeit schon grösstenteils ausgeglichen worden sein.

Tabelle 4.

Glykogen- und Fettgehalt der Leber der einfach mit einer kleineren Adrenalinmenge behandelten Kaninchen. 12 Stunden. nach $0,2 \mathrm{ccm}$ Adrenalin pro $\mathrm{kg}$.

5.-6. Febr. 1937.

\begin{tabular}{c|c|c|c|c}
\hline $\begin{array}{c}\text { Versuchstier- } \\
\text { Nr. }\end{array}$ & $\begin{array}{c}\text { Körpergewicht } \\
(\mathrm{g})\end{array}$ & \multicolumn{3}{|c}{ Leber } \\
\cline { 3 - 5 } & & Gewicht (g) & Glykogen (\%) & Fett (\%) \\
\hline 18 & 1870 & 58 & 1,86 & 2,98 \\
19 & 1680 & 49 & 1,71 & 3,41 \\
20 & 1690 & 40 & 1,56 & 2,65 \\
Durchschnitt & & & $\mathbf{1 , 7 1}$ & $\mathbf{3 , 0 1}$
\end{tabular}


Tabelle 5 .

Derselbe Tersuch wie oben mit einer mittelgrossen Adrenalinmenge. 12 Stunden nach 0,5 cem Adrenalin pro $\mathrm{kg}$.

1.-3. Febr. 1937.

\begin{tabular}{c|c|c|c|c}
\hline $\begin{array}{c}\text { Versuehstier- } \\
\text { Nr. }\end{array}$ & $\begin{array}{c}\text { Körpergewicht } \\
(\mathrm{g})\end{array}$ & \multicolumn{3}{|c}{ Leber } \\
\cline { 3 - 5 } & & Gewicht (g) & Glykogen (\%) & Fett $(\%)$ \\
\hline 21 & 1710 & 48 & 1,38 & 3,47 \\
22 & 1540 & 53 & 1,56 & 2,81 \\
23 & 1960 & 63 & 1,61 & 3,20 \\
Durohschnitt & & & $\mathbf{1 , 5 1}$ & $\mathbf{3 , 1 6}$
\end{tabular}

Tabelle 6.

Derselbe Versuch wie oben mit einer grösseren Adrenalinmenge.

12 Stunden nach $1 \mathrm{cem}$ Adrenalin pro kg.

20.-21. Dez. 1936.

\begin{tabular}{|c|c|c|c|c|}
\hline \multirow{2}{*}{$\begin{array}{c}\text { Versuchstier- } \\
\mathrm{Nr} \text {. }\end{array}$} & \multirow{2}{*}{$\begin{array}{c}\text { Körpergewicht } \\
(\mathbf{g})\end{array}$} & \multicolumn{3}{|c|}{ Leber } \\
\hline & & Gewicht (g) & Glykogen (\%) & Fett $(\%)$ \\
\hline $\begin{array}{c}24 \\
25 \\
26 \\
\text { Durchschnitt }\end{array}$ & $\begin{array}{l}1600 \\
1700 \\
1760\end{array}$ & $\begin{array}{l}39 \\
65 \\
58\end{array}$ & $\begin{array}{l}1,25 \\
1,00 \\
1,03 \\
1,09\end{array}$ & $\begin{array}{l}3,42 \\
3,02 \\
3,21 \\
3,22\end{array}$ \\
\hline
\end{tabular}

Im Gegensatz dazu fand man 12 Stunden nach Anwendung von $1,0 \mathrm{ccm}$ Adrenalinlösung den Glykogengehalt von 1,09\%, der also nach der anfänglichen Senkung noch nicht seinen Normalwert erreichte wahrscheinlich deshalb, weil einerseits die Glykogenausschüttung entsprechend der vermehrten Giftdosis recht tiefgreifend gewesen war, was schon Aoki in dieser Klinik experimentell nachwies, und anderseits der Ernährungszustand der seit 24 Stunden gehungerten Tiere sonst doch kleine Defekte der Glykogendepots unschwer decken konnte, aber grössere auszugleichen, wozu es natürlich eines grösseren Vorrates an Muttersubstanzen des Glykogens bedurfte, ihm nicht immer leicht zugänglich war. Unter diesen Umständen kann die Verabreichung der Substanzen, deren Geeignetsein zur Glokogenbildung zu prüfen ist, sehr treffend sein, da die Tierkörper dann jede mögliche Muttersubstanz des Glykogens begehren. Zur Hervorhebung der daraus aufgebauten Glykogenausbeute kann dabei einerseits die genügende Evakuation des von früher her dagewesenen Vorratglykogens immer vorteilhaft sein, weil der neugebildete Anteil dadurch klarer dargestellt wird, 
anderseits eine möglichst grosse Dosis des den Zuckerstoffwechsel anregrenden Giftstoffes auch dazu beitragen, weil je mehr Adrenalin dabei gebraucht, desto ausschlaggebender die Antwort ist, wie man aus den folgenden Versuchen ersieht. Leider tötet zu viel Adrenalin die Versuchstiere früher als der Versuchsschluss und in diesem Verhältnis scheint 1,0 ccm Adrenalin pro $\mathrm{kg}$ eine optimale Dose darzustellen, da die Tiere 1,5 ccm Adrenalin manchmal nicht überstehen. Der Fettgehalt der Leber wird unter Adrenalinwirkung im Gegensatz zu Glykogen vermehrt. Die Schwankungen sind aber überhaupt nicht so umfangreich wie die des Glykogens, wahrscheinlich wird das dabei in die Leber hineingeströmte Fett mehr oder minder reichlich in Glykogen umgewandelt, worauf später noch näher eingegangen wird.

\section{d) Glykogenbildung aus Fettsubstanzen unter Adrenalinwirkung.}

Aus den oben erwähnten Grunden wurden in diesen Versuchen alle 9 Tiere mit 1,0 cem Adrenalinlösung vorbehandelt, und erhielten damn weiter $9 \mathrm{~g}$ Olivenöl pro $\mathrm{kg}$ durch Sonde in den Magen. 3 (Tab. 7), 6 (Tab. 8) resp. 9 Stunden (Tab. 9) nach der Ölfütterung wurden je 3 Tiere sukzessiv abgetötet, sämtlich entsprechend 12 Stunden nach der Adrenalineinspritzung. Der dabei gefundene Glykogengehalt der Leber war resp. 1,12\% für 3-Stunden-, 2,26\% für 6-Stunden- und $1,79 \%$ für 9-Stundenversuch. Wenn man diese Werte mit 1,09\%, dem nach alleiniger Anwendung von 1,0 ccm Adrenalin gefundenen Glykogenwert vergleicht, so bemerkt man beim 3-Stundenversuch fast keinen Unterschied, wohl aber beim 6- und 9-Stundenversuch eine deutliche Vermehrung, und insbes. beim mittleren eine um 2 fach

Tabelle. 7 .

Glykogen-und Fettgehalt der Leber der Adrenalintiere zu verschiedenen Zeiten nach Fettfütterung. Adrenalin $1 \mathrm{ccm}$ u. Olivenöl $9 \mathrm{~g}$ pro $\mathrm{kg}$.

Tiere 3 Stunden nach Fettfütterung getötet.

15.--16. März 1937.

\begin{tabular}{|c|c|c|c|c|}
\hline \multirow{2}{*}{$\begin{array}{l}\text { Versuchstier- } \\
\text { Nr. }\end{array}$} & \multirow{2}{*}{$\begin{array}{c}\text { Körpergewieht } \\
(g)\end{array}$} & \multicolumn{3}{|c|}{ Leber } \\
\hline & & Gewicht (g) & Glykogen $(\%)$ & Fett $(\%)$ \\
\hline $\begin{array}{c}97 \\
28 \\
29 \\
\text { Durehsehnit }\end{array}$ & $\begin{array}{l}1890 \\
2100 \\
1700\end{array}$ & $\begin{array}{l}52 \\
61 \\
48\end{array}$ & $\begin{array}{l}1,21 \\
1,04 \\
1,11 \\
1,12\end{array}$ & $\begin{array}{l}3,47 \\
3,42 \\
3,25 \\
\mathbf{3 , 3 8}\end{array}$ \\
\hline
\end{tabular}


Tabelle 8.

Derselbe Versuch wie oben. Adrenalin $1 \mathrm{com} u$. Olivenöl $9 \mathrm{~g}$ pro $\mathrm{kg}$.

Tiere 6 Stunden nach Felffütterung getötet.

19.-20. Febr. 1937.

\begin{tabular}{c|c|c|c|c}
\hline $\begin{array}{c}\text { Versuchstier- } \\
\text { Nr. }\end{array}$ & $\begin{array}{c}\text { Körpergewicht } \\
(\mathrm{g})\end{array}$ & \multicolumn{3}{|c}{ Leber } \\
\cline { 3 - 5 } & & Gewicht (g) & Glykogen (\%) & Fett (\%) \\
\hline & & & & \\
30 & 1730 & 53 & 1,94 & 3,31 \\
31 & 1620 & 49 & 2,08 & $\mathbf{3 , 4 7}$ \\
32 & 1840 & 63 & 2,76 & 3,12 \\
Durchschnitt & & & $\mathbf{2 , 2 6}$ & $\mathbf{3 , 3 0}$
\end{tabular}

Tabelle 9.

Derselbe Versuch wie oben. Adrenalin $1 \mathrm{ccm} u$. Olivenöl $9 \mathrm{~g}$ pro $\mathrm{kg}$.

Tiere 9 Stunden nach Fettfütterung getötet.

17.-18. März 1937.

\begin{tabular}{c|c|c|c|c}
\hline \multirow{2}{*}{$\begin{array}{c}\text { Versuchstier- } \\
\text { Nr. }\end{array}$} & $\begin{array}{c}\text { Körpergewicht } \\
(\mathbf{g})\end{array}$ & \multicolumn{3}{|c}{ Leber } \\
\cline { 3 - 5 } & & Gewicht (g) & Glykogen (\%) & Fett (\%) \\
\hline 33 & 1680 & 47 & 1,78 & 3,61 \\
34 & 1900 & 52 & 1,69 & 3,28 \\
35 & 1570 & 54 & $\mathbf{1 , 9 0}$ & $\mathbf{3 , 8 5}$ \\
Durchschnitt & & & $\mathbf{1 , 7 9}$ & $\mathbf{3 , 5 8}$
\end{tabular}

so grosse, wobei der gesamte Glykogengehalt um 36\% grösser war als der Normalwert (=1,66\%). Was den Fettgehalt der Leber betrifft, so beobachtet man bei diesen Versuchsserien mit der Zeit zunehmende Steigerungen, die stärker als beim Versuch mit einfacher Adrenalinzufuhr (Tab, 6) sind, was man vielleicht auf das Einwandern des resorbierten Fettes nach Bedarf seitens der notleidenden Glykogendepots zurückführen kann. Die oben geschilderte stärkere Vermehrung des Leberglykogens bei gleichzeitiger Anwendung des Adrenalins und Fettes im Vergleich zur einfachen Adrenalinzufuhr deutet ungezwungen auf die Glykogenbildung aus gegebenem Fett unter der Adrenalinwirkung. Aus dem vorangehenden Versuch (Tab. 1) ergab sich schon, dass die Resorption des Fettes 3-5 Stunden nach der Einverleibung am lebhaftesten vor sich geht. Durch diesen Versuch (Tab. 7) wurde weiter festgestellt, dass 3 Stunden nach Fettgabe die Fettablagerung in der Leber schon eintritt, der Glykogengehalt sich aber noch nicht vermehrt, welch letzterer 6 Stunden nach Fett am stärksten und 9 Stunden danach wieder viel schwächer nachweisbar ist. Der Fettgehalt der Leber 6 Stunden nach Fettgabe war trotz der noch lebhaft 
vor sich gehenden Fettresorption gar nicht vermehrt im Vergleich zu 3 Stunden nach Fett, wohl deshalb, weil das eingeströmte Fett reichlich in Glykogen umgewandelt.wurde.

Die Verkleinerung des Glykogens 9 Stunden nach Fett als früher erklärt sich dadurch, dass um diese Zeit das entstandene Glykogen schon teilweise wieder verbraucht wurde, dessen Ersatz nunmehr wegen Mangel des vom Darm aus resorbierten Fettes ungenügend wurde. Jedenfalls geht aus diesem Versuch hervor, dass der Übergang des Fettes in Glykogen 6 Stunden nach der Fütterung am deutlichsten nachweisbar ist, weswegen in den nachfolgenden Versuchen die Tiere stets um diese Zeit getötet wurden. In Versuch 10 und 11, die bei variabler Adrenalinmenge den Einfluss eines konstanten Fettquantums auf die Glykogendepots zeigen sollten, wurden die Tiere aus den oben geschilderten Gründen stets entsprechend 12 Stunden nach Adrenalin und zwar 6 Stunden nach Abgabe von $9 \mathrm{~g}$ Olivenöl getötet. Beim Gebrauch von $0,2 \mathrm{ccm}$ Adrenalinlösung fand man den Glykogengehalt

$$
\text { Tabelle } 10 .
$$

Glykogen- und Fettgehalt der Leber der Kaninehen nach Fettfütterung bei

Behandlung mit variablen Adrenalinmengen. Tiere 12 Stunden nach $0,2 \mathrm{cem}$ Adrenalin und zugleich 6 Stunden nach $9 \mathrm{~g}$ Olivenöl getötet.

21.-22. Febr. 1937.

\begin{tabular}{c|c|c|c|c}
\hline $\begin{array}{c}\text { Versuchstier- } \\
\text { Nr. }\end{array}$ & $\begin{array}{c}\text { Körpergewicht } \\
(\mathbf{g})\end{array}$ & \multicolumn{3}{|c|}{ Leber } \\
\cline { 3 - 5 } & & Gewicht (g) & Glykogen (\%) & Fett (\%) \\
\hline 36 & 1520 & 37 & 1,88 & 3,41 \\
37 & 1880 & 49 & 1,92 & 3,57 \\
38 & 1660 & 40 & 1,79 & 3,39 \\
Durchschnitt & & & 1,86 & $\mathbf{3 , 4 6}$
\end{tabular}

Tabelle 11.

Derselbe Versuch wie oben mit ciner mittelgrossen Adrenalinmenge. 12 Stunden nach 0,5 ecm Adrenalin und zugleich 6 Stunden nach $9 \mathrm{~g}$ Olivenöl getötet.

20.-21. Febr. 1937.

\begin{tabular}{c|c|c|c|c}
\hline $\begin{array}{c}\text { Versuchstier- } \\
\text { Nr. }\end{array}$ & $\begin{array}{c}\text { Körpergewieht } \\
(\mathbf{g})\end{array}$ & \multicolumn{3}{|c|}{ Leber } \\
\cline { 3 - 4 } & & Gewicht $(\mathbf{g})$ & Glykogen $(\%)$ & Fett (\%) \\
\hline 39 & 1430 & $\mathbf{3 4}$ & $\mathbf{2 , 2 8}$ & $\mathbf{3 , 1 9}$ \\
40 & 1450 & $\mathbf{5 0}$ & $\mathbf{1 , 8 8}$ & $\mathbf{3 , 8 0}$ \\
$\mathbf{4 1}$ & 1680 & $\mathbf{4 6}$ & $\mathbf{2 , 1 6}$ & $\mathbf{3 , 5 6}$ \\
Durchsehnitt & & & $\mathbf{2 , 1 0}$ & $\mathbf{3 , 5 1}$
\end{tabular}


der Leber von 1,86\%, der sich von dem bei alleiniger Anwendung von der gleichen Menge Adrenalin (=1,71\%) nicht wesentlich unterschied, dagegen beim Versuch mit 0,5 ccm Adrenalin betrug der Glykogengehalt $2,10 \%$, also $39 \%$ mehr im Vergleich mit dem zugehörigen Kontrollwert von $1,51 \%$. Was die nach Fettfütterung stattfindende Zunahme des Glykogengehaltes beim Gebrauch von $1,0 \mathrm{ccm}$ Adrenalin betrifft, so ergab sich schon aus Versuch 8 der Betrag von 1,07\%, also ein noch viel grösserer als bei Versuch mit $0,5 \mathrm{ccm}$ Adrenalin. Der Fettgehalt der Leber wurde in diesen Versuchen im Vergleich zu den ohrie Fettfütterung tatsächlich vermehrt, aber nur leicht, zumal in geringerem Umfang als bei alleiniger Fettfütterung ohne Adrenalinanwendung (vgl. Versuch 15). Dieses Defizit an Fettzunahme kann natürlich mit der Glykogenbildung aus Fett unter Adrenalinwirkung in direktem Zusammenhang stehen.

Aus dem Geschilderten geht hervor, dass die Vermehrung der Glykogendepots nach Fettfütterung erst bei Anwendung einer verhältnismässig grossen Menge Adrenalin nachweisbar ist. Die Wirkung einer kleinen Adrenalinmenge muss sicher zu schwach sein, eine nachweisbare Glykogenausbeute auszulösen.

\section{c) Glykogenbildung aus Fettsäure unter Adrenalinwirkung.}

Nun gehe ich auf die Frage der Glykogenbildung aus freien Fettsäuren ein, die wie eingangs erörtert ein noch ungelöstes Rätsel des intermediären Stoffwechsels darstellt. Bekanntlich gibt es in der Literatur Arbeiten, die die Glykogenbildung aus Neutralfetten nachweisen konnten, wobei aber die Fettsäuren- wie schon gesagt nicht so leicht wie die Glyzerinkomponenten als Glykogenbildner anzunehmen sind. Auch fehlt es nicht an Untersuchungen, die die Glykogenbildung aus niederen Fettsäuren feststellten. So fanden Joseph und Hallman $^{38)}$ bei 2 Tage gehungerten Ratten nach peroraler Gabe von verschiedenen tieferen Fettsäuren eine deutliche Vermehrung des Leberglykogens. Stö $\mathrm{r}^{39}$ ) konnte bei gehungerten Ratten durch Fütterung mit Buttersäure und Kohlehydraten mehr Glykogenablagerung als durch alleinige Kohlehydratnahrung erzielen. Dasgleiche fand auch Eckstein ${ }^{40}$ bei Untersuchung mit Propionsäure. Im Gegensatz zu den tieferen ist die Glykogenbildung aus den hochwertigen Fettsäuren bisher noch nicht einwandfrei erwiesen. Die alte Untersuchung von Seegen ${ }^{41)}$ mit Leberbrei und Fettsäuren ist zu primitiv. Die neue 
Angabe von Tangl ${ }^{122}$ über Beobachtung von Vermehrung der ungesättigten Fettsäuren im Blut bei Pankreashunden nach Insulin, wodurch er den Utbergang der Fettsäuren in Zucker festgestellt zu haben glaubt, ist umständlich und nicht beweiskräftig genug. Gomi ${ }^{43)}$ konnte in dieser Klinik bei den durch Thyroxin vorbehandelten Tieren Neubildung von Glykogen aus peroral gegebenen Fettsäuren direkt nachweisen.

Zur Untersuchung wurden die Tiere wie üblich mit der durch Rindergallebeimischung emulgierter Oleinsäure in Menge von $5 \mathrm{~g}$ pro $\mathrm{kg}$ und dazu noch mit resp. $0,2,0,5$ und $1,0 \mathrm{ccm}$ Adrenalinlösung behandelt und sämtlich entsprechend 12 Stunden nach Adrenalin und zwar 6 Stunden nach Fettsäure getötet. Die Ergebnisse lauten wie folgt: $0,2 \mathrm{ccm}$ Adrenalin verursacht in diesem Falle ebenso wenig Veränderungen des Glykogenvorrates wie beim Versuch mit Neutralfett (Tab. 12). Bei Anwendung von 0,5 ccm Adrenalin gewinnt man das

Tabelle 12.

Gtykogen- und Fettgehalt der Leber der mit Oleinsäure gefütterten Kaninchen bei Behandlung mit einer lleineren Adrenalinmenge. 12 Stunden nach $0,2 \mathrm{ccm}$ Adrenalin und 6 Stunden naeh

$5 \mathrm{~g}$ Oleinsäure pro $\mathrm{kg}$ getölet.

4.-5. Febr. 1937.

\begin{tabular}{c|c|c|c|c}
\hline $\begin{array}{c}\text { Versuchstier- } \\
\text { Nr. }\end{array}$ & $\begin{array}{c}\text { Körpergewicht } \\
(\mathrm{g})\end{array}$ & \multicolumn{3}{|c}{ Leber } \\
\cline { 3 - 5 } & & Gewicht (g) & Glykogen (\%) & Fett (\%) \\
\hline 42 & 2130 & $\mathbf{7 8}$ & 1,92 & \\
43 & 1780 & 43 & 2,06 & 3,67 \\
44 & 1730 & 52 & 1,60 & 3,43 \\
Durchschnitt & & & 1,86 & $\mathbf{3 , 4 0}$ \\
\end{tabular}

Tabelle 13.

Derselbe Versuch wie oben mit einer mittelgrossen Adrenalinmenge. 12 Stunden nach 0,5 cem Adrenalin pro $\mathrm{kg}$ und.

6 Stunden nach Oleinsäure getötet.

28.-29. Jan. 1937.

\begin{tabular}{|c|c|c|c|c|}
\hline \multirow{2}{*}{$\begin{array}{l}\text { Versuchstier- } \\
\text { Nr. }\end{array}$} & \multirow{2}{*}{$\begin{array}{c}\text { Körpergewicht } \\
(\mathrm{g})\end{array}$} & \multicolumn{3}{|c|}{ Leber } \\
\hline & & Gewicht (g) & Glykogen $(\%)$ & Fett $(\%)$ \\
\hline $\begin{array}{c}45 \\
46 \\
47 \\
\text { Durchschnitt }\end{array}$ & $\begin{array}{l}1780 \\
1680 \\
1580\end{array}$ & $\begin{array}{l}54 \\
52 \\
52\end{array}$ & $\begin{array}{l}1,89 \\
1,72 \\
2,04 \\
1,88\end{array}$ & $\begin{array}{l}3,51 \\
3,24 \\
3,21 \\
3,32\end{array}$ \\
\hline
\end{tabular}


Leberglykogen von 1,88\% (Tab. 13), das im Vergleich zum Kontrollwert von alleiniger Adrenalinzufuhr (vgl. Tab. 5) um 24,5\% vermehrt wird. $1 \mathrm{ecm}$ Adrenalin bringt 1,94\% Glykogengehalt der Leber hervor (Tab. 14), der freilich um 78\% grösser als die zugehörige Kontrollzahl (vgl. Tab. 6) ist. Durch Verabreichung der hohen Fettsäuren kann man also der Adrenalinwirkung unterliegenden Tiere einen recht reichlichen Zuschlag des Leberglykogens erzielen. Der Fettgehalt der Leber vermehrt sich auch in diesen Versuchen nachweisbar, was wahrscheinlich aus der aus dem Darm resorbierten Fettsäure herrührt, wobei die Grösse der vermehrten Fettmenge umgekehrt proportional zu der verwendeten Adrenalinmenge steht. Ein ähnliches Verhalten beobachtete man schon bei den Versuchen mit Neutralfett.

Beiläufig erwähne ich an dieser Stelle den Glykogen- und Fettgehalt der Leber der allein mit Olivenöl resp. Oleinsäure behandelten Tiere ; die Ergebnisse geben die Kontrollwerte für die vorangehenden Versuche (Tab. 15 u. 16). Man fand dabei fast keine Abweichung des

Tabelle 14.

Derselbe Versuch wie oben mit einer grösseren Adrenalinmenge.

12 Stunden nach $1 \mathrm{ccm}$ Adrenalin pro $\mathrm{kg}$ und 6 Stunden naeh Oleinsäure getötet.

14. - -15. Dez. 1936.

\begin{tabular}{c|c|c|c|c}
\hline \multirow{2}{*}{$\begin{array}{c}\text { Versuchstier- } \\
\text { Nr. }\end{array}$} & $\begin{array}{c}\text { Körpergewicht } \\
(\mathrm{g})\end{array}$ & \multicolumn{3}{|c|}{ Leber } \\
\cline { 3 - 5 } & & Gewicht (g) & Glykogen (\%) & Fett $(\%)$ \\
\hline & & & 1,87 & \\
48 & 1600 & 50 & $\mathbf{2 , 0 3}$ & $\mathbf{3 , 9 9}$ \\
49 & 1460 & $\mathbf{4 4}$ & 1,91 & $\mathbf{2 , 5 2}$ \\
50 & 1800 & & $\mathbf{1 , 9 4}$ & $\mathbf{2 , 9 7}$ \\
Durchschnitt & & &
\end{tabular}

Tabelle 15.

Glykogen- und Fettgehält der Leber der bloss mit Olivenöl gefütterten Kananinchen. 6 Stunden nach Fütterung mit $9 \mathrm{~g}$ Olivenöl pro $\mathrm{kg}$ getötet.

25.-26. Febr..1937.

\begin{tabular}{c|c|c|c|c}
\hline $\begin{array}{c}\text { Versuchstier- } \\
\text { Nr. }\end{array}$ & $\begin{array}{c}\text { Körpergewicht } \\
(\mathbf{g})\end{array}$ & \multicolumn{3}{|c}{ Leber } \\
\cline { 2 - 4 } & & Gewicht $(\mathbf{g})$ & Glykogen $(\%)$ & Fett (\%) \\
\hline 51 & 1870 & 58 & 1,62 & 3,56 \\
52 & 1710 & 52 & 1,84 & 4,10 \\
53 & 1900 & 62 & 1,64 & 3,87 \\
Durchschnitt & & & $\mathbf{1 , 7 0}$ & $\mathbf{3 , 8 5}$
\end{tabular}


Tabelle 16. Glykogen-und Fettgehalt der Leber der mit Oleinsöure gefütterten Kaninchen. 6 Stunden nach Fütterung mit $5 \mathrm{~g}$ Oleinsäure pro $\mathrm{kg}$ getötet.

22.--23. Jan. 1937.

\begin{tabular}{c|c|c|c|c}
\hline $\begin{array}{c}\text { Versuchstier- } \\
\text { Nr. }\end{array}$ & $\begin{array}{c}\text { Körpergewicht } \\
(\mathbf{g})\end{array}$ & \multicolumn{3}{|c}{ Leber } \\
\cline { 3 - 5 } & & Gewieht $(\mathrm{g})$ & Glykogen (\%) & Fett (\%) \\
\hline & $\mathbf{1 6 3 0}$ & $\mathbf{5 0}$ & 1,87 & \\
54 & 1720 & 48 & 1,60 & 3,79 \\
55 & 1760 & 58 & $\mathbf{1 , 4 8}$ & 3,56 \\
56 & & & $\mathbf{1 , 6 5}$ & $\mathbf{3 , 6 2}$ \\
Durehschnitt & & &
\end{tabular}

Glykogengehaltes von der Norm, dagegen einen mehr oder minder grösseren Fettgehalt als bei gleichzeitiger Wirkung von Adrenalin. Daraus ergibt sich, dass die Fettstoffe erst dann in der Leber in Glykogen umgewandelt werden, wenn die Tierkörper durch Adrenalinwirkung zum gereizten Kohlehydratstoffwechsel veranlasst werden. Das Streben der Leber unter Adrenalin nämlich ist, aus allen möglichen Quellen Glykogen neuzubilden, und dies dann als Zucker wieder auszuschütten.

\section{f) Glykogenbestand der glykogenberaubten Leber unter Adrenalinwirkung.}

Im vorangehenden habe ich die Versuche mit den Tieren angestellt, deren Leber noch eine ansehnliche Menge Vorratglykogen besass. In den ähnlichen Arbeiten früherer Autoren wurde das Vorratglykogen ausgangs durch verschiedene Mittel, wie langfristige Karenz, forcierte einseitige Fett- oder Eiweissnahrung, Strychninkrämpfe und endlich Phloridzinvergiftung, möglichst aus den Depots evakuiert, wodurch die Glykogenneubildung begünstigt, oder wenigstens deren Erkennen wesentlich erleichtert werden kann. Aus diesem Grunde habe ich vor allem die in dieser Klinik von $\mathrm{O}$ to mo und $\mathrm{Naga}^{44}$ ausgearbeitete Lezithinmethode bevorzugt, die darin besteht, dass man den Kaninchen pro $\mathrm{kg} 10$ ccm einer $10 \%$ iger Lezithinaufschwemmung intravenös injiziert, wodurch während der Zeitfrist von 6-24 Stunden nach der Injektion das Vorratglykogen aus der Leber der wie gewöhnlich gefütterten Tiere vollständig ausgeschwemmt wird, ohne dabei das Organparenchym allzu sehr zu schädigen. Tabelle 17 gibt die Analysenwerte der Leber der Kaninchen wieder, die 18 Stunden nach der oben geschilderten Lezithinbehandlung getötet wurden. Das 
Leberglykogen erwies sich als Null, was den Befund von Otom o und Naga o bestätigt. Einen ebenso geringen Glykogengehalt fand man in der Leber der Tiere, die in gleicher Weise mit Lezithin behandelt wurden und dazu noch Neutralfett (Tab. 18) resp. Fettsäure (Tab. 19)

Tabelle 17.

Veränderung des Glykogen- und Fettgehaltes der Leber dureh Lezithininfusion. Tiere 18 Stunden nach Lezithin getötet.

6.-8. Jan. 1937.

\begin{tabular}{c|c|c|c|c}
\hline \multirow{2}{*}{$\begin{array}{c}\text { Versuchstier- } \\
\text { Nr. }\end{array}$} & $\begin{array}{c}\text { Körpergewicht } \\
(\mathbf{g})\end{array}$ & \multicolumn{3}{|c|}{ Leber } \\
\cline { 3 - 5 } & & Gewicht (g) & Glykogen (\%) & Fett (\%) \\
\hline 57 & 1700 & 57 & 0 & 3,57 \\
58 & 1580 & 38 & 0 & 3,72 \\
59 & 1870 & 54 & 0 & 3,84 \\
Durchschnitt & & & $\mathbf{3 , 7 1}$
\end{tabular}

Tabelle 18.

Glykogen- und Fettgehalt der Leber der mit Lezithin vorbehandelten und danach mit Olivenöl gefütterten Kaninchen. Tiere 18 Stunden nach Lezithin und zugleich 6 Stunden nach Olivenöl getötet. Olivenöl pro $\mathrm{kg} 9 \mathrm{~g}$.

15.-17. Jan. 1937.

\begin{tabular}{c|c|c|c|c}
\hline $\begin{array}{c}\text { Versuehstier- } \\
\text { Nr. }\end{array}$ & $\begin{array}{c}\text { Körpergewicht } \\
(\mathbf{g})\end{array}$ & \multicolumn{3}{|c}{ Leber } \\
\cline { 3 - 5 } & & Gewicht (g) & Glykogen (\%) & Fett (\%) \\
\hline 60 & 1670 & 48 & 0 & $\mathbf{4 , 7 6}$ \\
$\mathbf{6 1}$ & 1640 & $\mathbf{5 6}$ & 0 & $\mathbf{4 , 1 3}$ \\
$\mathbf{6 2}$ & 2140 & 76 & 0 & $\mathbf{3 , 9 2}$ \\
Durchschnitt & & & $\mathbf{4 , 2 7}$
\end{tabular}

Tabelle 19 .

Derselbe Versuch wie oben, aber mit Oleinsäure statt Olivenöls. Tiere 18 Stunden nach Lezithin und zuglcich 6 Stunden nach Oleinsäure getötct. Oleinsäure pro $\mathrm{kg} 5 \mathrm{~g}$.

17.--19. Jan. 1937.

\begin{tabular}{c|c|c|c|c}
\hline $\begin{array}{c}\text { Versuchstier- } \\
\text { Nr. }\end{array}$ & $\begin{array}{c}\text { Körpergewicht } \\
(\mathrm{g})\end{array}$ & \multicolumn{3}{|c|}{ Leber } \\
\cline { 3 - 5 } & & Gewicht $(\mathrm{g})$ & Glykogen (\%) & Fett $(\%)$ \\
\hline & & & & \\
63 & 1690 & 52 & 0 & $4 ; 56$ \\
64 & 1870 & 58 & 0 & 4,10 \\
65 & 1900 & 61 & 0 & $\mathbf{3 , 7 0}$ \\
Durchschnitt & & & 0 & $\mathbf{4 , 1 2}$
\end{tabular}


erhielten, wobei aber natürlich eine deutliche Fettanhäufung stattfand. Lezithininfusion und Fettnahrung sind ebenfalls Mittel, die Glykogenausschwemmung hervorzurufen; es ist kein Wunder, dass das Zusammenwirken beider Faktoren eine vollständige Glykogenevakuation zur Folge hatte. In Versuch 20 und 21 wurden die Tiere zunächst mit Lezithin und 6 Stunden danach noch mit 1,0 resp. $0,5 \mathrm{ccm}$ Adrenalin behandelt und dann nach weiteren 12 Stunden getötet. Die Glykogenausbeute betrug resp. 0,61 und $0,39 \%$, und in soviel Mengen muss man die Neubildung des Glykogens durch Adrenalin erkennen, denn das Vorratglykogen war dabei durch Lezithinbehandlung schon vollständig ausgeschwemmt worden. Das hierbei gefundene Glykogen darf man allerdings nicht ohne weiteres als aus Lezithin umgewandeltes betrachten, vielleicht geht die Glykogenbildung aus gewöhnlichen Muttersubstanzen in der glykogenleeren Leber lebhafter als sonst vor sich, wenn auch der Utbergang des Lezithins in Glykogen in einem zwangmässig gereizten Stoffwechselzustand wie unter Adrenalin wirkung keineswegs auszuschliessen ist. Diese Möglichkeit, die

Tabelle 20.

Glykogen- und Fettgehalt der Leber bei Zusammenwirken von Adrenalin und

Lezithin. Tiere 18 Stunden nach Iezithin und zugleich 12 Stunden nach Adrenalin getötet. Adrenalin pro $\mathrm{kg} 1 \mathrm{ccm}$.

29.-31. Dez. 1936.

\begin{tabular}{c|c|c|c|c}
\hline $\begin{array}{c}\text { Versuchstier- } \\
\text { Nr. }\end{array}$ & $\begin{array}{c}\text { Körpergewieht } \\
(\mathrm{g})\end{array}$ & \multicolumn{3}{|c}{ Leber } \\
\cline { 3 - 4 } & & Gewicht $(\mathrm{g})$ & Glykogen $(\%)$ & Fett (\%ó) \\
\hline & 1730 & 49 & 0,76 & 2,92 \\
66 & 1740 & 54 & 0,64 & 3,45 \\
68 & 1710 & 52 & 0,44 & 2,54 \\
Durchschnitt & & & $\mathbf{0 , 6 1}$ & $\mathbf{2 , 9 7}$
\end{tabular}

Tabelle 21.

Derselbe Versuch wie oben mit einer kleineren Adrenalinmeng. $0,5 \mathrm{ccm}$ Adrenalin pro $\mathrm{kg}$.

8.-10. Febr. 1937.

\begin{tabular}{c|c|c|c|c}
\hline \multirow{2}{*}{$\begin{array}{c}\text { Versuchstier- } \\
\text { Nr. }\end{array}$} & $\begin{array}{c}\text { Körpergewicht } \\
(\mathrm{g})\end{array}$ & \multicolumn{3}{|c|}{ Leber } \\
\cline { 3 - 5 } & & Gewicht $(\mathrm{g})$ & Glykogen $(\%)$ & Fett $(\%)$ \\
\hline & 1640 & 45 & 0 & \\
70 & 1450 & 50 & 0,68 & $\mathbf{3 , 4 7}$ \\
71 & 1560 & 38 & 0,49 & $\mathbf{3 , 4 1}$ \\
Durchschnitt & & & $\mathbf{0 , 3 9}$ & $\mathbf{3 , 6 7}$ \\
\end{tabular}


vielleicht dasselbe Schicksal wie die Frage der Zuckerbildung aus Fettsüuren teilt, ist noch nicht als sichergestellt erwiesen, obwohl einige dafür sprechende Beobachtungen schon vorliegen. Best und Hershe $y^{45}$. fanden z. B. bei Pankreashunden vermehrte Zuckerausscheidung nach Lezithinfütterung. $\mathrm{Iz}_{\text {a }}{ }^{46)}$ konnte in einem ähnlichen Versuch auch Blutzuckersteigerung zeigen. Einer endgültigen Feststellung harren jedoch noch weitere Untersuchungen.

\section{g). Glykogenbildung aus Fettstoffen in der glykogenleeren Leber unter Adrenalinwirkung.}

Im vorigen Versuch habe ich schon festgestellt, dass Adrenalin die durch Lezithinbehandlung glykogenleer gemachte Leber zum Wiederaufbau einer wenn auch kleinen Menge Glykogen veranlassen kann. In diesem Versuch wurde weiter untersucht, ob die Glykogenausbeute dabei durch perorale Zufuhr von Fett resp. Fettsäure sich vermehren könne. In letzterem Falle kann man noch einen sicheren Anhaltspunkt für die Zuckerbildung aus Fettstoffen gewinnen.

Zur Untersuchung wurden die Tiere, wie in den vorangehenden Versuchen dieser Arbeit die Regel ist; 24 Stunden hungern gelassen, obwohl die glykogenausschwemmende Wirkung von Lezithininfusion auch bei gewöhnlicher Fütterung ebenso gut vor sich geht. Das Hungern schadet der Glykogenbildung nicht, sondern befördert sie, was von Pollak, Junkersdorf ${ }^{47}$, Ohara u. a. angenommen wurde, die zwar erst nach langfristiger Karenz den Übergang des Fettes in

\section{Tabelle 22.}

Glykogen- und Fettgehalt der Leber der mit Lezithin vorbehandelten und danach mit Olivenöl gefütterten Kaninchen unter Einwirkung van Adrenalin.

Tiere 18 Stunden nach Lezithin, zugleich 12 Stunden nach Adrenalin und 6 Stunden nach Olivenöl getötet. Adrenalin $1 \mathrm{ccm}$, Olivenöl $9 \mathrm{~g}$ pro $\mathrm{kg}$.

6.-8. März 1937.

\begin{tabular}{c|c|c|c|c}
\hline $\begin{array}{c}\text { Versuchstier- } \\
\text { Nr. }\end{array}$ & $\begin{array}{c}\text { Körpergewicht } \\
(\mathrm{g})\end{array}$ & \multicolumn{3}{|c}{ Leber } \\
\cline { 3 - 5 } & & Gewicht (g) & Glykogen (\%) & Fett (\%) \\
\hline & 1870 & 62 & 1,89 & $\mathbf{3 , 0 1}$ \\
$\mathbf{7 2}$ & 1930 & 45 & 2,04 & $\mathbf{2 , 8 9}$ \\
$\mathbf{7 4}$ & 1860 & 47 & $\mathbf{2 , 8 2}$ & $\mathbf{2 , 9 9}$ \\
Durchschnitt & & & $\mathbf{2 , 2 5}$ & $\mathbf{2 , 9 6}$
\end{tabular}


Kohlehydrate nachweisen konnten. Die übrigen Versuchsanordnungen gestalteten sich wie üblich, nämlich die Tiere, die vor 18 Stunden mit Lezithin und vor 12 Stunden mit Adrenalin behandelt worden waren, wurden 6 Stunden nach der Fettfütterung getötet. Tabelle 2225 geben die Ergebnisse dieser Versuche wieder. Durch perorale Gabe von Olivenöl gewannen die mit $1,0 \mathrm{ccm}$ Adrenalin behandelten Lezithintiere (Tab. 22) eine Vermehrung an Leberglykogen um 268\% im Vergleich zu den Tieren mit dergleichen Vorbehandlungen (vgl. Tab. 20). In einem anderen Versuch (Tab. 23) fand man durch Gabe von Oleinsäure statt Olivenöl eine um $226 \%$ betragende Glykogenvermehrung. Bei Anwendung einer halben Dose Adrenalin war der Glykogengehalt der Leber viel niedriger und betrug bei den mit Olivenöl gefütterten Lezithintieren $1,12 \%$ (Tab. 24) und bei den mit Oleinsäure gefütterten $0,83 \%$ (Tab. 25); diese Zahlen sind noch viel grösser als der zugehörige Kontrollwert (=0,39\%, vgl. Tab.21). Der Fettgehalt der Leber zeigte in diesen Versuchen keine grossen Schwankungen, allenfalls eine eben nachweisbare Abnahme im Vergleich zu den Tieren, die ohne Adrenalin sonst gleich vorbehandelt wurden (vgl. Tab. 18 u. 19).

Tabelle 23.

Derselbe Versuch wie oben, aber mit Oleinsäure statt Olivenöls. Adrenalin 1ecm, Oleinsäure $5 \mathrm{~g}$ pro $\mathrm{kg}$.

22.-24. Dez. 1936.

\begin{tabular}{c|c|c|c|c}
\hline $\begin{array}{c}\text { Versuchstier- } \\
\text { Nr. }\end{array}$ & $\begin{array}{r}\text { Körpergewicht } \\
(\mathrm{g})\end{array}$ & \multicolumn{3}{|c}{ Leber } \\
\cline { 3 - 5 } & Gewicht (g) & Glykogen (\%) & Fett (\%) \\
\hline 75 & 1720 & 53 & 1,64 & 2,71 \\
76 & 2200 & 66 & $\mathbf{2 , 5 6}$ & 3,06 \\
77 & 1760 & 49 & $\mathbf{1 , 7 6}$ & 2,16 \\
Durchsehnitt & & & $\mathbf{1 , 9 9}$ & $\mathbf{2 , 6 4}$
\end{tabular}

Tabelle 24.

Derselbe Versuch wie oben mit Olivenil, aber bei einer mittelgrossen Adrenalinmenge. Adrenalin $0,5 \mathrm{ccm}$, Olivenöl $9 \mathrm{~g}$ pro $\mathrm{kg}$.

12.-14. März 1937.

\begin{tabular}{c|c|c|c|c}
\hline $\begin{array}{c}\text { Versuchstier- } \\
\text { Nr. }\end{array}$ & $\begin{array}{c}\text { Körpergewicht } \\
(\mathbf{g})\end{array}$ & \multicolumn{3}{|c}{ Leber } \\
\cline { 3 - 5 } & Gewicht (g) & Glykogen (\%) & Fett (\%) \\
\hline 78 & 1700 & 55 & 1,28 & $\mathbf{3 , 5 1}$ \\
79 & 1850 & 55 & 1,38 & $\mathbf{2 , 8 7}$ \\
80 & 1600 & 48 & 0,70 & 3,13 \\
Durchschnitt & & & $\mathbf{1 , 1 2}$ & $\mathbf{3 , 1 7}$
\end{tabular}


Tabelle 25.

Derselbe Fersuch wie oben mit Oleinsüure: Adrenalin $0,5 \mathrm{~cm}$, Oleinsäure $5 \mathrm{~g}$ pro $\mathrm{kg}$.

6.-8. Febr. 1937.

\begin{tabular}{c|c|c|c|c}
\hline $\begin{array}{c}\text { Versuchstier- } \\
\text { Nr. }\end{array}$ & $\begin{array}{c}\text { Körpergewicht } \\
(\mathrm{g})\end{array}$ & \multicolumn{3}{|c}{ Leber } \\
\cline { 3 - 5 } & & Gewicht (g) & Glykogen (\%) & Fett (\%) \\
\hline 81 & 1520 & 53 & & \\
82 & 1720 & 43 & 0,76 & 3,12 \\
883 & 1670 & 49 & 0,71 & 3,23 \\
Durchschnitt & & & $\mathbf{0 , 8 3}$ & $\mathbf{3 , 0 5}$ \\
\hline
\end{tabular}

IV. Besprechung der Ergebnisse.

Alles in allem genommen ist durch vorliegende Arbeit festgestellt, dass man durch Fütterung mit Fettstoffen die Leber der unter Adrenalinwirkung sich befindenden Tiere leicht zur Anhäufung einer grossen Menge von Vorratglykogen zwingen kann. Die auf diese Weise erzielte Vermehrung des Glykogengehaltes beträgt bei gewöhnlichen Tieren 107\% nach Neutralfett- und 78\% nach Fettsäurefütterung, sie kann sich sogar bei den Tieren, deren Leber im vorảus glykogenleer gemacht war, auf $268 \%$ nach jener und $226 \%$ nach dieser steigern. Der so angeschwollene Glykogenvorrat überwiegt gewöhnlich den Standardwert der gar nicht vorbehandelten Tiere mehr oder minder stark. Das lässt sich leicht dadurch erklären, dass der Kohlehydratstoff wechsel der Tiere durch Einwirkung von Adrenalin zwangmässig gesteigert wird, so dass er wie folgt vorsichgeht: gesteigerte Glykogenbildung aus allermöglichen Muttersubstanzen-ebenso gesteigerte Ausschüttung des Vorrat- sowie neugebildeten Glykogens-Hyperglykämie, mitunter auch Glykosurie, gesteigerte Zuckerverbrennung in der Peripherie. Welcher von diesen 3 Vorgängen dem direkten Angriffspunkt des Adrenalins entspricht, lässt sich nicht so ohne weiteres sagen. ${ }^{48)}$ Jedenfalls kann der eine die anderen als notwendige Folge hervorrufen und in der Tat bestehen 3 Vorgänge gleichzeitig, vielleicht aber nicht immer in gleicher Stärke, sondern deren Wirkungsoptima folgen zeitweise ziemlich nacheinander. So können die Depots zu den Zeitabschnitten an Glykogen verlieren, in denen der Dissimilationsvorgang den Assimilations- überwiegt, und vice versa. Im Laufe der Adrenalinwirkung gibt es freilich eine besondere Reaktionsphase, in der die Glykogenausschüttung schon grösstenteils abgeklungen war, 
doch die lebhafte Glykogenbildung noch immer fortbesteht, die in der vorliegenden Arbeit zur Untersuchung der Fettstoffe als Kohlehydratbildner benutzt wurde. Der Nachweis einer kolossalen Anhäufung des Glykogens, womit die Tierkörper in derartigen Phase auf die Fitterung der Fettstoffe reagierten, weist ungezwungen darauf hin, dass die Kohlehydrate aus den gefütterten Substanzen gebildet werden. Aus dem Befund, dass dabei die Fütterung der freien Fettsäuren auch eine deutliche Glykogenanhäufung verursachte, kann man vielleicht mit Recht die Genese der Kohlehydrate auch aus hohen Fettsäuren annehmen, dessen Möglichkeit festzustellen das Hauptthema dieser Arbeit ist.

\section{Zusammenfassung.}

1. Adrenalin verursacht in der Leber der normalen Kaninchen eine Abnahme des Vorratglykogens, in der im voraus glykogenleer gemachten Leber dagegen Anhäufung einer ansehnlichen Menge von Glykogen.

2. Die Glykogendepots der normalen Kaninchen erfahren unter Wirkung von Adrenalin doch eine deutliche Zunahme statt Abnahme, wenn Fettstoffe zu' rechter Zeit eingetroffen sind. Die Leber gewinnt dabei an Glykogen um ca. 100\%.

3. Eine derartige Glykogenanhäufung in der Leber der Adrenalintiere nach Fettfütterungen vollzieht sich durch vorläufige Evakuation des Vorratglykogens noch stärker. Der Glykogengehalt vermehrt sich dadurch um ca. $200 \%$.

4. Bei den genannten Glykogenansatz auslösenden Fettfütterungen kommen nicht nur Neutralfette, sondern auch freie Fettsäuren in Betracht. Letztere wirken dabei als Glykogenbildner fast ebenso stark wie erstere.

5. Die Glykogenbildung aus Fettstoffen setzt um so stärker ein, je grössere Menge Adrenalin dabei mit wirkt.

\section{Literatur.}

(1) Asher u. Clavo-Criad o, Biochem. Ztschr., 1925, 164, 76.

(2) Bickenbach u. Junkersdorf, Areh. f. exper. Pathol. u. Pharmakol, $1928,132,129$.

(3) B l u m, Arch. f. d. ges. Physiol., 1902, 90, 617.

(4) Velieb, Virchow's Arch., 1906, 184, 345.

(5) Eppinger, Falta u. Rudinger, Ztschr. f. klin. Med., 1908, 66, 1.

(6) Wertheimer, Arch. f. d. ges. Physiol., 1926, 213, 287. 
(7) Polla k, Arch. f. exper. Pathol. u. Pharmakol., 1909, 61, 166.

(8) Roubitschek, Arch. f. d. ges. Physiol, 1914, 155, 68.

(9) Burn u. Marks, Journ. of Physiol, 1926, 61, 497.

(10) Oh a ra, Tohoku Journ. of Exper. Med., 1925, 6, 1.

(11) M atsui, Nagasaki Igakkai Zasshi, 1930, 8, 1178.

(12) A oki, Tohoku Journ. of Exper. Med., 1936, 29, 244.

(13) V. Fürth, Lehrbuch der physiologischen und pathologischen Chemie, Leipzig 1927, II., 223.

(14) Kimu r a, Tohoku Igaku Zasshi, 1935, I8, 481.

(15) K u magawa u. Su to, Biochem. Ztschr., 1908, 8, 212. 43,38 .

(16) London u. Wersilowa, Jahresber. Fortschr. Tierchemie (Maly), 1913,

(17) Arnshink, Ztsehr. f. Biol., 1890, 26, 434.

(18) Levites, Ztsohr. f. physiol. Chem., 1907, 53, 349.

(19) Munk u. Rosenstein, Arch. f. Anat. u. Physiol., Physiol. Abt., 1890, 376.

(20) Strack, Versuche über ganz einseitige Ernährung, Leipzig 1932, 204.

(21) Pflüger, Arch. f. d. ges. Physiol., 1900, 82, 303; 1901, 86, 1.

(22) S a to, Tohoku Journ. of Exper, Med., 1923, 4, 265.

(23) Doy on u. Ka reff, Compt. rend. Soc. Biol., 1904, 56, 66 ,

(24) Wol ownik, Virchow's Arch., 1905, 180, 225.

(25) Bang, Der Blntzucker, Wiesbaden 1913, 89.

(26) C. F. Cori u. G. T. Cori, Proc. Soc. Exper, Biol. \& Med., 1928, 25, 258.

(27) Geiger u. Schmidt, Arch. f. exper. Pathol, u. Pharmakol., 1929, 143, 321.

(28) Corkill u. Marks, Journ. of Physiol., 1930, 70,67.

(29) Bierry u. Gruzews k a, Compt. rend. Acad. Sciens. 1906, 142, 1165.

(30) Molitor, Arch. f. exper. Pathol. u. Pharmakol, 1930, 154, 280.

(31) C. F. Cori u. G. T. Cori, Jonrn. of Biol. Chem., 1929, 85, 275.

(32) S a c k s, Amer. Journ. of Physiol., 1931, 97, 557.

(33) Young, Journ. of Physiol., 1931, 73, 103.

(34) S a hy un u. Luek, Journ. of Biol. Chem., 1929, 85, 1 .

(35) G. T. Cori, C. F. Cori u. Buchwald, Journ: of Biol. Chem., 1930, 86, 375

(36) N a k ats u k a, Kyoto Furitsu Ikadaigaku Zasshi, 1932, 6, 1351.

(37) L öw u. Pfeile r, Biochem. Ztschr., 1928, 193, 278.

(38) Jose ph u. Hall m an, Proc. Soc. Exper. Biol. \& Med., 1935, 32, 1351.

(39) Stör, Ztschr. f. physiol. Chem., 1933, 220, 27.

(40) Eckstein, Journ. of Biol. Chem,, 1933, 102, 591.

(41) Seege n, Arch. f. d. ges. Physiol., 1886, 39, 132.

(42) Tangl, Biochem. Ztschr., 1931, 241, 87.

(43) Go mi, Tohoku Igaku Zasshi, 1936, 19, 1005.

(44) Ot o mo u. Naga o, Tohoku Journ. of Exper. Med., 1935, 25, 63.

(45) Best a. Hershey, Journ. of Physiol., 1932, 75, 49.

(46) I z a r, Riforma Med., 1929, 75, 49. Zit nach Kongresszbl. f. d. ges. inn. Med., $1930,55,221$.

(47) Junkersdorf, Arch. f. d. ges. Physiol., 1921, 186, 238; 1922, 197, 500 ; $1923,200,443 ; 1925,207,433 ; 1925,208,617$.

(48) Wiesel, Bethe u. Bergmanns Handbuch der normalen und pathologischen Physiologie, Berlin 1930, XVI/1,526. 